BIOBUSINESS BRIEFS

MARKET WATCH

\section{Evolution of Chinese bioclusters as a framework for investment policies in emerging markets}

Stimulating life sciences innovation is an important policy issue for governments in emerging markets (Nature Rev. Drug Discov. 13, 646-647; 2014; Nature Biotech. 31, 195-201; 2013). This article analyses biomedical clusters in China to understand the evolution of these ecosystems and to propose a framework that could help guide government policy decisions in other emerging markets. China is the only emerging market that has attempted to build multiple bioclusters, and so could potentially provide the most robust data for such an analysis.

To develop the framework, data indicating the intensity of two types of activity entrepreneurial activity and academic activity - were collected for eight Chinese bioclusters. Entrepreneurial activity was assessed through the number of venture-backed biotech companies and contract research organizations, and academic activity was assessed using the number of publication citations and rankings of academic institutions; see Supplementary information S1 (box) for details. Each biocluster was then assigned to a quadrant of a $2 \times 2$ matrix based on the intensity of entrepreneurial and academic activity within them (FIG. 1a).

Applying this framework to other emerging markets could help guide local government policies and investment decisions (FIG. 1b), depending on which of the four broad categories - 'innovation leaders', 'untapped talent', 'niche hubs' or 'aspiring' the bioclusters seem to most closely resemble.
For innovation leaders, government policy should be to invest to strengthen such ecosystems. The approach needs to be multipronged: increased funding for academic institutions, stronger incentives for start-ups, investments in research and development (R\&D) infrastructure and talent development. In addition to Shanghai and Beijing, other clusters in emerging markets that are well positioned to become innovation leaders - if supported by sustained investment policies - include Tel Aviv, Seoul, Bangalore and Singapore.

For clusters in the untapped talent group, there is a need to nurture science coming out of academic labs and to translate it into products, and government policies should aim to 'unlock' potential. This can be accomplished by strengthening key aspects such as incubators to facilitate start-ups, project and early-stage venture financing, and management talent. Suzhou became a world leader in the medical devices area by providing the companies and academics in its BioBay Park with access to lab space, core equipment facilities, development of business plans and funding networks. Wuhan's BioLake Park is now emulating many of these principles to tap its potential. St Petersburg, Istanbul, Kolkata, São Paulo and Mexico City are examples from emerging markets that fall into this category.

'Niche hubs' can be built as centres of excellence; for example, clinical trial hubs in Warsaw or information technology/digital
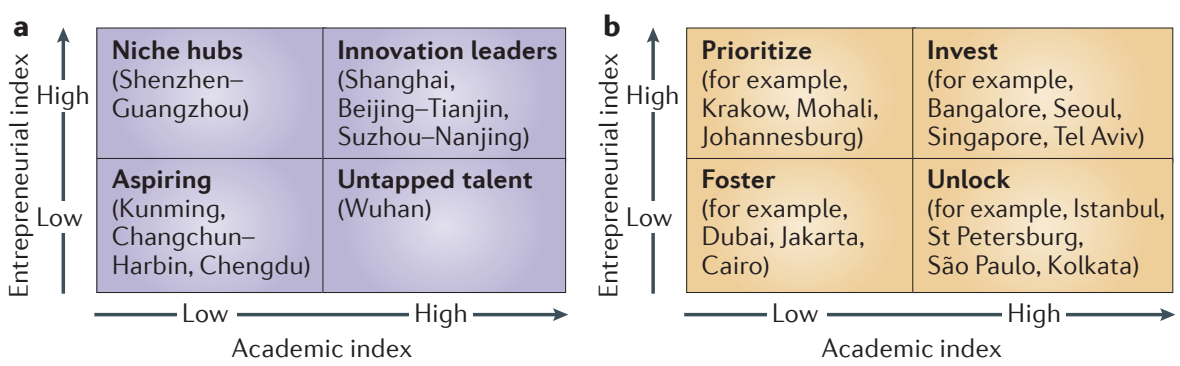

Figure 1 |A framework for investment policies in emerging markets. a | Entrepreneurial and academic indices were used to assign eight Chinese bioclusters in the $2 \times 2$ matrix, based on whether the values for that biocluster were above or below the median value for the group of bioclusters overall. b | Investment policies framework. See Supplementary information S1 (box) for details. health hubs in Gurgaon. Government policy should be to 'prioritize' the strengthening of these hubs before transitioning them towards becoming innovation leaders. The Shenzhen cluster is now a global leader in bioinformatics, owing to strong government funding and support that helped create BGI, the leading company in this space, which leveraged Shenzhen's traditional strengths in computational sciences, electronics and informatics.

Aspiring bioclusters require long-term planning driven by investments in strengthening academic and scientific centres. One key reason why Dubai lags behind in life sciences innovation in spite of strong funding and being a well connected, global trading hub is the lack of a strong university base a hurdle that Singapore successfully overcame by fostering leading institutions such as the National University of Singapore.

This framework is not intended to provide a precise strategy, and there are limitations of applying the centralized investment model of China to other emerging markets. Nevertheless, it can be an additional tool for policy makers to strategically evaluate their clusters to inform investment decisions and policies, and to serve as a guide for industry decisions on potential locations for new research units. For example, with regard to India's recent decision to invest in three bioclusters - Bangalore, Mohali and Faridabad - the model indicates that Bangalore would be most effectively supported through an 'invest' strategy as an innovation leader and Mohali through a 'prioritize' strategy to strengthen its leadership position as a niche hub in agri-food biotechnology. However, an 'unlock' strategy could be more effective for an untapped talent cluster such as Kolkata, which has a high academic intensity (described in more detail in Supplementary information S1 (box)).

Many such decisions are no doubt influenced by regional politics. However, if the governments in emerging markets harbour ambitions of building life sciences innovation hubs such as Boston, San Francisco and Cambridge, UK, in their countries, their investment decisions and policies need to be more strategic.

Ajay Gautam is Executive Director and Head of Collaborations, AsiaPac \& Emerging Markets, IMED Biotech Unit at AstraZeneca, Shanghai, China. e-mail:ajay.gautam@astrazeneca.com The author declares no competing interests. 